\title{
Generation of resonant Alfvén waves in the auroral oval
}

\author{
V. A. Pilipenko ${ }^{1,2}$, D. Yu. Klimushkin ${ }^{3}$, P. N. Mager $^{3}$, M. J. Engebretson ${ }^{4}$, and O. V. Kozyreva ${ }^{1,2}$ \\ ${ }^{1}$ Institute of Physics of the Earth, 123242 Moscow, Russia \\ ${ }^{2}$ Space Research Institute, 117997 Moscow, Russia \\ ${ }^{3}$ Institute of Solar-Terrestrial Physics, 664033 Irkutsk, Russia \\ ${ }^{4}$ Augsburg College, Minneapolis, MN 55454, USA \\ Correspondence to: V. A. Pilipenko (pilipenk@ augsburg.edu)
}

Received: 7 July 2015 - Revised: 27 December 2015 - Accepted: 19 January 2016 - Published: 16 February 2016

\begin{abstract}
Many independent observations have shown that resonant field line Pc5 pulsations (typical periods of about several minutes) are preferably excited inside the auroral oval. Consideration of the most evident interpretation schemes shows that there is no simple explanation of this effect. Here we consider theoretically the generation of toroidal Pc5 Alfvén waves by fluctuating magnetospheric field-aligned currents. It is shown that the Alfvén wave latitudinal structure has the same features as in the case of the classical field line resonance driven by external sources: a peak localized in latitude and a $180^{\circ}$ phase shift across the resonant magnetic shell. This model suggests an additional mechanism of ultra-low-frequency (ULF) wave excitation which can operate at auroral latitudes.
\end{abstract}

Keywords. Magnetospheric physics (auroral phenomena; current systems; MHD waves and instabilities)

\section{Introduction}

One of the key notions of ultra-low-frequency (ULF) wave physics in planetary magnetosphere is the field line Alfvén resonance, suggested by Tamao (1965), Chen and Hasegawa (1974), Radoski (1974), and Southwood (1974). The original field line resonance theory was formulated in the framework of a simple geometry with straight field lines. Since then the model has been advanced to more complicated 2-D (Fedorov et al., 1998; Leonovich and Mazur, 1989) and 3-D (Cheng, 2003) geometry, but the key notions have remained the same. According to this theory when a monochromatic source in the outer magnetosphere drives the magnetosphere, standing Alfvén waves in the magnetospheric resonator, terminated by the conjugate ionospheres, are excited. The most intense response occurs at the resonant magnetic shell where the source frequency matches the local eigenfrequency of the magnetospheric resonator. Though the source and Alfvén resonant shell are separated by an opaque region for the fast mode, thanks to the large scale of low- $m$ modes, significant wave energy is transported inward via this mechanism from the outer magnetosphere.

These theories have successfully explained the majority of ground and spacecraft measurements of amplitude-phasepolarization latitudinal structure. The standard field line resonance theory predicts a latitudinal wave structure with a local amplitude maximum and phase gradient at a resonant shell. The phase gradient typically corresponds to an apparent poleward propagation. This specific amplitude-phase structure is considered as an irrefutable argument in favor of excitation of low- $m$ ULF waves by external sources such as the Kelvin-Helmholtz instability (KHI), or periodic buffeting of the magnetopause by the solar wind dynamic pressure. Statistical studies indeed show a high correlation between Pc5 wave power (typical frequencies about $2-7 \mathrm{mHz}$ ) and the solar wind parameters such as a flow speed and dynamic pressure (e.g. Engebretson et al., 1998; Mann et al., 1999; Potapov, 2013). Some case studies have tracked an energy flow from magnetopause fluctuations to Alfvén waves on ground, as predicted by the field line resonance theory (Rae et al., 2005, 2007; Agapitov et al., 2009; Hartinger et al., 2011).

According to this theory, the location of the resonant $L$ shell is determined by the distribution of the Alfvénic field line period - that is, mass plasma density and magnetic field - in the magnetosphere, and it should not depend strongly on the ionospheric conductance, or field-aligned current (FAC). However, a number of observations have indicated 
that the location of the auroral oval is a preferable latitude for magnetospheric resonator excitation: namely Raspopov and Afanasieva (1982) noticed that the morning Pc5 generation region was predominantly located near the eastern edge of the westward auroral electrojet, near the equatorial boundary of the statistical position of the auroral oval; Lam and Rostoker (1978) and later Pilipenko et al. (2001) showed that the positions of the westward auroral electrojet and the Pc5 wave power are closely linked to each other, and during their meandering with time the position of the Pc5 latitudinal peak remained within the borders of the auroral electrojet; Lepidi and Francia (2003), analyzing a statistical latitudinal distribution of low-frequency $(1-4 \mathrm{mHz})$ geomagnetic fluctuations, concluded that the latitude of their maximum power could be considered as a marker of the auroral oval position; Simms et al. (2006) showed statistically that the movement of the auroral oval correlated with Pc5 spectral power independent of changes in the solar wind and interplanetary magnetic field (IMF); and a spatial association of magnetospheric Pc5 waves with FACs was reported by Potemra et al. (1988) and Bochev et al. (2009). Thus, Pc5 wave activity turned out to be closely coupled with auroral electrodynamics, comprising the ionospheric electrojet, magnetospheric FACs, and auroral particle precipitation. Nonetheless, this effect has not been taken into account by modern theories of ULF waves, though it could be significant for a substantial augmentation of existing Pc5 models.

Here we suggest that Pc5 resonant structure can be excited by a local source, in particular, by fluctuations of the FAC inside the auroral oval. We discuss how the high-latitude Pc5 observations comply with theoretical notions of field line Alfvén resonance.

\section{Model of local excitation of field line Alfvén resonance}

A characteristic feature of the auroral oval is the presence of intense FACs which drive the auroral electrojet and provide energy for aurora activation. The steady part of the FAC $j_{0}$ creates a shear of magnetic field lines. This shear can cause a transverse dispersion of small-scale (high- $m$ ) poloidal Alfvén waves (Klimushkin and Mager, 2004), but it is not significant for large-scale toroidal Alfvén waves.

The FAC inevitably has a fluctuating (non-steady) part. The FAC's fluctuations can have many causes: intensification of the global magnetosphere-ionosphere current system due to injections of energetic particles in the magnetosphere (Saka et al., 1992), buffeting of the magnetosphere by the solar wind and/or magnetosheath fluctuations (Kozlovsky et al., 1994), filamentation and pinching of FAC (Galperin et al., 1986), magnetotail activity, etc. Indeed, analysis of magnetic fluctuations detected during the 1.5 years of the low-orbiting DE-2 satellite mission clearly demonstrated that statistical contours of classical FAC are perfectly consistent with the areas of turbulence with scale ranging from a few kilometers to several hundred kilometer occurrence (Golovchanskaya et al., 2006). These and other (e.g., Ohtani et al., 1996; Golovchanskaya and Maltsev, 2004) observations confirmed that magnetospheric FACs, both downward and upward region R1, are inherently turbulent. The fluctuating part of FAC is assumed here to be a driver of the toroidal Alfvén waves.

We present a theoretical discussion that supports the conjecture of a broadband source excitation of toroidal (low- $m$ ) Alfvénic structure in a finite region. As a mathematical basis, we use the classical box model of the magnetosphere with straight homogeneous magnetic field (Southwood, 1974). In this model the $x$ (radial) coordinate is directed across the magnetic shells, the $z$ (parallel) coordinate is directed along field lines, and the $y$ (azimuthal) coordinate is directed across both field lines and magnetic shells. Plasma density $\rho(x)$ varies only in the radial direction, imitating the latitudinal dependence of the Alfvén eigenperiod.

Combination of the linearized equation for small plasma oscillations, Ampere's law with a fluctuating external current $\boldsymbol{j}$, Faraday's law, and the frozen-in condition gives an equation for the wave electric field (Klimushkin et al., 2012):

$$
\frac{1}{V_{\mathrm{A}}^{2}} \frac{\partial^{2} \boldsymbol{E}}{\partial t^{2}}-\nabla \times \nabla \times \boldsymbol{E}=-\frac{4 \pi}{c^{2}} \frac{\partial \boldsymbol{j}}{\partial t},
$$

where $V_{\mathrm{A}}=B / \sqrt{4 \pi \rho}$ is the Alfvén speed. Fluctuations of the current $\boldsymbol{j}(t)$ are assumed to be a driver of magnetohydrodynamic (MHD) waves (the right-hand term in Eq. 1).

The two-dimensional wave electric field can be expressed as a sum of the potential and rotational components $\boldsymbol{E}=$ $-\nabla_{\perp} \Phi+\nabla_{\perp} \times \boldsymbol{e}_{z} \Psi$, where $\boldsymbol{e}_{z}=\boldsymbol{B} / \boldsymbol{B}$ is the unit vector along the ambient magnetic field $\boldsymbol{B}$, and $\nabla_{\perp}=\left(\partial_{x}, i k_{y}\right)$ is the transverse Hamilton operator. The potentials $\Phi$ and $\Psi$ represent the electric field of the Alfvén wave mode and fast mode, respectively (Klimushkin, 1994; Itonaga et al., 2000). In this paper we consider the case of Alfvén mode excitation by a FAC; therefore the fast-mode contribution $\propto \Psi$ is neglected. The transverse electric field $E$ of the Alfvén mode can be characterized by the potential $\Phi$, as follows: $\boldsymbol{E}=-\nabla_{\perp} \Phi$. The corresponding magnetic component of the Alfvén wave is determined by $\partial_{t} \mathbf{B}=c\left[\boldsymbol{e}_{z} \times \nabla_{\perp}\right] \partial_{z} \Phi$. The Alfvén wave mode transports the transverse $\mathbf{j}_{\perp}$ and field-aligned $j_{z}$ currents, namely $\mathbf{j}_{\perp}=\Sigma_{\mathrm{A}} V_{\mathrm{A}}^{-1} \partial_{t} \mathbf{E}_{\perp}$ and $j_{z}=\Sigma_{\mathrm{A}} \nabla_{\perp}^{2} \Phi$, where $\Sigma_{\mathrm{A}}=c^{2} / 4 \pi V_{\mathrm{A}}$ is the Alfvén wave conductance.

In the box model the dependence on the time and the azimuthal and parallel coordinates can be presented in the form $\exp \left(-i \omega t+i k_{y} y+i k_{z} z\right)$. Then, deriving $\boldsymbol{E}$ via the $\Phi$ potential Eq. (1) has been transformed into an equation for the Alfvénic mode in a 1-D inhomogeneous plasma:

$$
\frac{\partial}{\partial x}\left[k_{\mathrm{A}}^{2}(\omega, x)-k_{z}^{2}\right] \frac{\partial \Phi}{\partial x}-k_{y}^{2}\left[k_{\mathrm{A}}^{2}(\omega, x)-k_{z}^{2}\right] \Phi=\widetilde{q}(x, \omega),
$$

where $k_{\mathrm{A}}(\omega, x) \equiv \omega / V_{\mathrm{A}}(x)$ is the Alfvénic wave number. 
In Eq. (2) the right-hand term $\widetilde{q}(x, \omega)$ is the spectrum of current fluctuations (the source term of the wave equation). Owing to the current continuity condition, $\nabla \cdot \boldsymbol{j}=0$, it can be expressed in terms of the current $j_{z}$ as

$\widetilde{q}(x, \omega)=\frac{4 \pi i \omega}{c^{2}}\left(\nabla_{\perp} \cdot j\right)=\frac{4 \pi \omega k_{z}}{c^{2}} j_{z}(x, \omega)$.

The boundary conditions for Eq. (2) on the radial and the parallel coordinates are $\left.\Phi\right|_{x \rightarrow \pm \infty}=0$ and $\left.\Phi\right|_{z=z_{ \pm}}=0$, where $z_{ \pm}$are the parallel coordinates of the field line conjugate points (ionospheres).

The Alfvénic resonance occurs on the coordinate $x_{\mathrm{R}}(\omega)$ determined as a solution of the equation $\omega=\omega_{\mathrm{A}}(x)=k_{z} V_{\mathrm{A}}(x)$, where $\omega_{\mathrm{A}}(x)$ is the magnetic shell eigenfrequency. Provided that a linear radial profile for the resonance frequency is assumed, $\omega_{\mathrm{A}}(x, \omega)=\Omega_{\mathrm{A}}(1-x / l)$, the coordinate of the resonance surface is expressed as

$x_{\mathrm{R}}(\omega)=l\left(1-\frac{\omega}{\Omega_{0}}\right)$.

Then, the wave Eq. (2) can be rewritten in the form

$\frac{\partial}{\partial x}\left[x-x_{\mathrm{R}}(\omega)\right] \frac{\partial \Phi}{\partial x}-k_{y}^{2}\left[x-x_{\mathrm{R}}(\omega)\right] \Phi=q(x)$,

where the source term is

$q(x, \omega)=2 \pi j_{z} \frac{V_{\mathrm{A}}^{2}}{c^{2}} \frac{k_{z} l}{\Omega_{\mathrm{A}}}$.

Equation (5) enables one to find a relationship between the level of the FAC fluctuations and the Alfvénic resonant response. Notice that a similar equation describes the resonant Alfvén wave excitation by an external fast mode (e.g., Leonovich, 2001). Singularity at $x \rightarrow x_{\mathrm{R}}(\omega)$ in Eq. (5) results in the growth of Alfvénic oscillations at a resonant shell under a permanent driving. In a realistic situation, this growth is terminated by dissipation in the system, accounted for by an imaginary correction term $x_{\mathrm{R}} \rightarrow x_{\mathrm{R}}+i \Delta$, where $\Delta$ is an effective semi-width of the resonant peak. Under a permanent driving the latitudinal scale $\Delta$ of a localized resonant peak is determined by the dominant dissipation factor. Commonly, this is assumed to be Joule dissipation in the ionosphere (Rae et al., 2007; Hartinger et al., 2015), but in the auroral oval more efficient specific dissipation mechanisms can operate, which will be enumerated below.

This type of equation can be considered for various forms of a $q(x)$ source. Hasegawa et al. (1983) predicted that a local field line can oscillate at its resonant frequency in response to a wide-band source. Klimushkin et al. (2012) solved this equation for a source localized at point $x=x_{0}$ distinct from the resonant point when $q(x)=\delta\left(x-x_{0}\right)$. Besides a localized resonant peak at $x=x_{\mathrm{R}}$, forced oscillations at $x=x_{0}$ are to be induced. Here we suppose that a source has a finite width with a characteristic scale $a$ and is described by the Gaussian function

$q=q_{0} \exp \left[-\frac{\left(x-x_{0}\right)^{2}}{2 a^{2}}\right]$

where $x_{0}$ is the location of the source maximum intensity, and $q_{0}$ is the maximum value of the source. The case $a \ll$ $\Delta$ corresponds to the model considered in Hasegawa et al. (1983) and Klimushkin et al. (2012). Here we consider the case $a \geq \Delta$.

A solution of Eq. (5) near the resonant point $x_{\mathrm{R}}$ can be written in integral form, following Mager and Klimushkin (2008):

$\Phi(x)=i \frac{q\left(x_{\mathrm{R}}\right)}{k_{y}} \int_{0}^{\infty} \frac{\mathrm{d} k}{\sqrt{k^{2}+k_{y}^{2}}} \exp \left[i k\left(x-x_{\mathrm{R}}\right)\right]$.

At the resonant point, the solution has a logarithmic singularity:

$\Phi(x)=-\frac{q_{0}}{k_{y}} \log \left(x-x_{\mathrm{R}}\right) \exp \left[-\frac{\left(x-x_{0}\right)^{2}}{2 a^{2}}\right]$.

The wave's electric azimuthal and magnetic radial components have a logarithmic singularity:

$E_{y}(x)=-i k_{y} \Phi=i q_{0} \log \left(x-x_{\mathrm{R}}\right) \exp \left[-\frac{\left(x-x_{0}\right)^{2}}{2 a^{2}}\right]$,

$B_{x}=\frac{c k_{z}}{\omega} E_{y}$,

whereas the radial electric and azimuthal magnetic components have a pole singularity

$E_{x}(x)=-\frac{\partial \Phi}{\partial x}=\frac{q_{0}}{k_{y}\left(x-x_{\mathrm{R}}\right)} \exp \left[-\frac{\left(x-x_{0}\right)^{2}}{2 a^{2}}\right]$,

$B_{y}=-\frac{c k_{z}}{\omega} E_{x}$.

The occurrence of this singularity produces a localized amplitude latitudinal peak of $E_{x}$ and $B_{y}$ components and a $180^{\circ}$ wave phase shift across the resonant magnetic shell just as in the case of external generation of the ULF wave in classical field line resonance theory. Thus, the occurrence of a poleward phase gradient associated with a localized resonant maximum does not necessarily indicate excitation of this structure by an external source.

The amplitude of the wave generated by the FAC is exponentially small unless the resonant shell is located inside the current, that is, at distances from the center of the current $\left|x_{\mathrm{R}}-x_{0}\right| \leq a$. Thus, the relevant Alfvén eigenfrequencies vary from $\omega_{\mathrm{A}}^{(1)}$ to $\omega_{\mathrm{A}}^{(2)}$, where $\omega_{\mathrm{A}}^{(1,2)} \equiv \omega_{\mathrm{A}}\left(x_{0} \pm a\right)$ are the Alfvén eigenfrequencies on the outer and the inner edges of the current, respectively (we assume the resonant frequency 


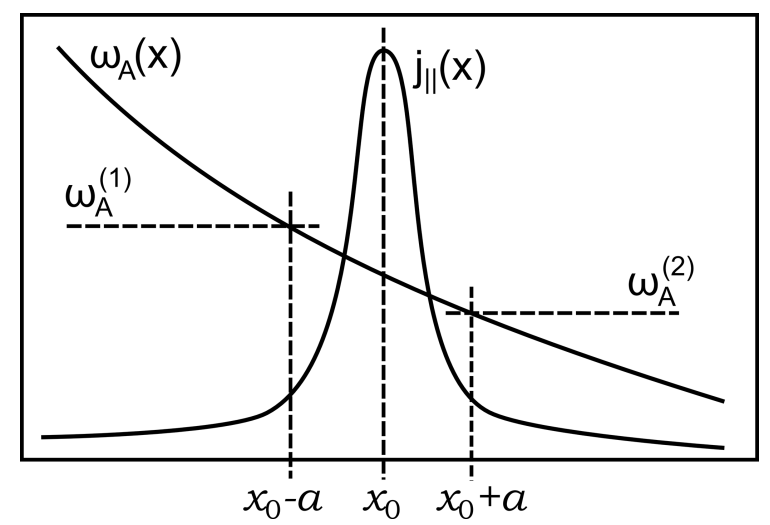

Figure 1. Range of the resonant frequencies determined by FAC location.

to decrease with the radial coordinate; see Fig. 1). Though FAC fluctuations are broadband, the resonant response of field lines inside the auroral oval selects narrowband Alfvén oscillations.

Let us estimate the level of FAC fluctuations $j_{\|}$necessary to produce the observed oscillations of the magnetic field toroidal component $B_{y}$. It is assumed that $\left|x-x_{\mathrm{R}}\right| \simeq \Delta$. From Eqs. $(6,12)$, one obtains by the order of magnitude (in SI units)

$B_{y} \simeq \mu_{0} j_{z} \frac{L_{\perp}^{2}}{2 m \Delta}$,

where $L_{\perp}$ is the transverse inhomogeneity characteristic scale in the magnetosphere. Let us suppose that near the equatorial plane of the magnetosphere $L_{\perp} \sim 1 R_{\mathrm{E}}, \Delta=$ $10^{3} \mathrm{~km}$, the azimuthal wave number $m=5$. Then to generate resonantly an Alfvén wave with amplitude $b_{y} \sim 50 \mathrm{nT}$ a fluctuating FAC is necessary with magnitude $j_{\|} \sim 10^{-8} \mathrm{~A} \mathrm{~m}^{-2}$. This estimate confirms that FAC fluctuations with realistic magnitudes indeed can stimulate Pc5 pulsations in the magnetosphere.

\section{Discussion: possible mechanisms for the Pc5 wave-auroral oval association}

The occurrence rate and intensity of Pc5 waves has a strong maximum in the morning sector and a weaker one in the afternoon sector. The azimuthal phase propagation and the polarization features of the ground magnetic disturbance in the horizontal plane change across the noon meridian (Samson, 1972). These observations led to the conclusion that KHI at the magnetopause or low-latitude boundary layer (LLBL) is a likely candidate for a Pc5 generation source (Walker, 1981; Fujita et al., 1996; Engebretson et al., 1998; Mann et al., 1999). Later, indications were found that impulsive or irregular variations of the dynamic pressure of the solar wind and magnetosheath constitute a possible source of Pc5 wave packets in the magnetosphere (Southwood and Kivelson, 1990; Walker, 2002; Kessel et al., 2004; Zolotukhina, 2010; Mazur, 2010; Potapov, 2013). Wave disturbances generated by these dayside sources propagate tailward along both sides of the magnetosphere, thus producing an azimuthal phase velocity pattern across the noon meridian. These mechanisms can work simultaneously: e.g., pressure-induced disturbance can be amplified by the KHI upon propagation along the magnetospheric flanks (e.g., Mazur and Chuiko, 2013).

Amplitude and phase latitudinal distributions of Pc5 waves clearly demonstrate resonance properties (Saka et al., 1982; Ziesolleck and McDiarmid, 1994), which are better seen in the morning hours. The amplitude maximum position depends on frequency (frequency increases towards low $L$ values). However, this feature may be obscured by peculiarities of the source spectrum, so amplitude-phase gradients are more robust indicators of resonant effects. The apparent meridional phase velocity in the vicinity of the amplitude maximum is to be directed poleward. The existence of resonance effects for Pc5 geomagnetic pulsations is commonly considered as an indicator of the wave energy transfer from an external source towards localized field line Alfvén oscillations (e.g., Menk and Waters, 2013).

From these notions on Pc5 generation, it follows that the ionospheric auroral current or auroral oval structure seemingly does not influence wave excitation. However, some observational results, listed in the Introduction section, indicate that in fact the auroral oval plays an active role in Pc5 excitation. Here, on the basis of existing knowledge of MHD wave physics, we consider the most evident interpretation schemes of auroral oval-Pc5 coupling.

The depression of magnetic field often preceding Pc5 activity in the early morning hours may indicate that the ULF wave generation mechanism is related to an energetic electron injection. Indeed, there are direct observations showing that morning time Pc5 pulsations might be associated with increased fluxes of energetic electrons in the magnetosphere (Saka et al., 1992). Nevertheless, difficulties emerge when interpreting morning time Pc5 pulsation behavior with this mechanism. Wave generation by energetic particles is most probably connected with kinetic instabilities. The small lateral scale (high $m$ values) of excited oscillations is a natural condition necessary for the effective interaction between low-frequency waves and energetic particles, because in this case the ratio of a Larmor radius to a lateral wavelength is finite (Pokhotelov et al., 2005; Klimushkin and Chen, 2006). Oscillations of this type are rarely recorded by groundbased magnetometers because high- $m$ oscillations are effectively screened by the ionosphere. However, the assumption that substorm-related morning Pc5 pulsations are generated by resonant instabilities of energetic electrons, similar to the excitation of storm-time Pc5 waves in the dusk sector by energetic protons, contradicts ground-based observations, which show low $m$ values and large lateral scales of these oscillations (Olson and Rostoker, 1978). A possibility 
for Alfvén wave generation by a non-steady transverse current of drifting energetic particles (the "ship waves" mechanism by Mager and Klimushkin, 2008) also results in the excitation of high- $m$ waves.

Rostoker and Lam (1978) proposed an interpretation of the electrojet-Pc5 relationship, suggesting that Pc5 waves are the eigenmodes of a 3-D magnetosphere-ionosphere current system. The electrical parameters of this circuit - i.e., resistance of the ionosphere, capacitance of the magnetospheric plasma flow, and inductance of FACs - determine the oscillation regime of the effective LRC circuit. However, the proposed 3-D current system oscillation cannot be considered as an alternative oscillatory system with a lower frequency than the Alfvén eigenfrequency, because in fact the eigenfrequency of this 3-D current system turns out to be the common Alfvén frequency $\omega_{\mathrm{A}}$ (Pilipenko et al., 2001).

The ionospheric conductivity in the region of the auroral oval is increased as compared with the background ionosphere, due to electron precipitation in the region of upward FAC. Observations of Sutcliffe and Rostoker (1979) demonstrated an increase of Pc5 pulsation strength across the dawn terminator (around 7.5 MLT), evidencing the important influence of ionospheric conductivity on Pc5 excitation. Therefore, it could be suggested that an increase of the ionospheric conductance, which occurs in the region of the auroral oval, would stimulate Pc5 activity. However, even background ionospheric conductance $\Sigma_{P} \simeq 5 \mathrm{~S}$ of the subauroral ionosphere is high enough as compared with the effective magnetospheric conductance $\Sigma_{\mathrm{A}} \simeq 1 \mathrm{~S}$. Moreover, a decrease of the ionospheric conductance $\Sigma_{P}$ during predawn hours is to be accompanied by a decrease of the magnetospheric plasma conductance $\Sigma_{\mathrm{A}}$, so the condition of good reflection, $\Sigma_{P} \gg \Sigma_{\mathrm{A}}$, is still kept. Thus, Alfvén wave reflection from the high-latitude ionosphere is good even at latitudes beyond the auroral oval and does not prevent the excitation of the magnetospheric Alfvén resonator.

Magnetic pulsations can be produced via the periodic modulation of precipitating particle fluxes. This idea was suggested for the interpretation of band-limited pulsations at dayside cusp latitudes (the "ionospheric transistor" mechanism of Engebretson et al., 1991) and in the auroral electrojet region (Leonovich, 2000; Saka et al., 2015). However, the problem of identifying a suitable periodic modulation factor still remains. Another difficulty for this interpretation exists: fluctuating east-west ionospheric currents should produce synchronous magnetic variations on the ground: the $H$ component is expected to be in phase along the meridian with a maximum beneath the auroral electrojet, while the $Z$ component must be out of phase at both sides from the ionospheric current, with a minimum beneath the electrojet. In fact, a closer examination of magnetograms shows that the $H$ and $Z$ components demonstrate apparent poleward phase propagation. This phase structure is consistent with the resonant model, based on the assumption of local excitation of the magnetospheric Alfvén resonator by an external driver, and contradicts the idea of modulated ionospheric current.

In principle, it might be suggested that the plasma, magnetic field, and field line geometry across the high-latitude magnetosphere vary in such a coordinated way that a resonant shell always happens to be inside the dynamic auroral oval. It seems that the proposed explanation is more natural. Parameters of the magnetospheric plasma 3-D distribution (including heavy ions) and magnetic field geometry are not so well known in the magnetosphere, especially at high latitudes. Therefore, even advanced modeling cannot indicate undoubtedly the location of field line resonance for a specific event. At the same time, observations have indicated that Pc5 power (both broadband and narrowband) is concentrated inside the auroral oval, but not elsewhere. We suppose that this fact must be incorporated into ULF physics theory, and this paper is a step in this direction.

\subsection{Pc5 wave excitation in the auroral oval during various phases of magnetic storms}

A characteristic feature of the auroral oval is the occurrence of a FAC system. These magnetospheric FACs, both downward and upward, are inherently turbulent. We have suggested that this feature is responsible for the close association between the location of the auroral oval and the epicenter of Pc5 wave activity. This hypothesis makes it possible to interpret from a unified standpoint the reported relationships between Pc5 wave activity and the auroral oval, auroral ionospheric electroject, and magnetospheric FACs. The idea that time-varying auroral FACs can drive MHD turbulence was suggested by Cerisier et al. (1987). Their theory predicted that a current pulse with velocity on the order of the Alfvén velocity can generate inertial Alfvén waves (a small-scale mode with a scale of about the plasma inertial scale $\left.\lambda_{e}=c / \omega_{e}\right)$. Our theoretical consideration has shown that upon driving of Alfvén waves by coherent FAC fluctuations the azimuthal component of the magnetic field has a pole singularity, and the radial component has a logarithmic singularity. This wave structure is typical for the toroidal polarization Alfvén field line oscillations driven by an external fast mode. Therefore, the Pc5 waves excited by fluctuations of auroral FACs are to have the same amplitude-phase latitudinal structure as classical resonant ULF waves.

Though the correspondence between the auroral oval and Pc5 wave "epicenter" may be considered just an accidental coincidence, which does not deserve any in-depth examination, we think that it is important to draw the attention of the magnetospheric community to this fact. ULF wave physics inside the auroral region are rather specific; therefore the standard field line resonance model, which was originally developed for midlatitudes, is to be augmented for this region. During the storm growth phase, the resonant response is to be severely suppressed by a high level of plasma and magnetic field variability. Fluctuations of the Alfvén resonator 
eigenfrequency can considerably decrease the quality factor of the resonator (Coult et al., 2007). As a result, only forced broadband magnetic fluctuations are to be expected during the storm/substorm main phase. During the recovery phase, the level of magnetospheric variability is much lower, so the resonant excitation of the magnetospheric Alfvén resonator becomes more efficient.

Another characteristic feature of auroral field lines is the occurrence of a non-resistive potential drop in the auroral acceleration region (AAR) produced by the mirror force. This force produces effective electric resistance accompanied by the dissipation of energy, though this energy does not dissipate locally but is transported by accelerated electrons from the magnetosphere into the ionosphere. During auroral activations the additional damping of Pc5 oscillations owing to the interaction with the AAR can overturn the Joule dissipation in the ionosphere and suppress Alfvén field line oscillations (Vogt and Haerendel, 1998; Fedorov et al., 2001). Nonetheless, in studies of Pc5 wave energy dissipation in the ionosphere only the Joule heating has been considered so far (Rae et al., 2007; Hartinger et al., 2015).

This proposed mechanism has something in common with suggestions about driving of the magnetospheric Alfvén resonator by oscillatory ionospheric currents, e.g., the ionospheric transistor model (Engebretson et al., 1991). It is possible that in a realistic magnetosphere a combination of several mechanisms of Pc5 wave excitation may take place. For example, Kelvin-Helmoltz disturbances generated during periods of high solar wind flow can provide a seed turbulent background in the magnetosphere, which further facilitates Alfvén wave excitation by auroral FACs.

Being a broadband source, FAC fluctuations generate a continuum of Alfvén waves with different frequencies located on resonant magnetic $L$ shells corresponding to frequencies $\omega_{\mathrm{A}}(L)$. FACs have a finite width across the $L$ shells. The resonant Alfvén waves located outside the FAC region will have vanishingly small amplitudes. Only the waves generated inside this region will have noticeable amplitudes. On the other hand, the resonant frequency profile in the magnetosphere depends only on the distribution of the magnetic field and mass density in the magnetosphere and (outside the plasmapause) decreases with the radial coordinate. The resonant frequencies corresponding to the FAC location are contained in a narrow range from, say, $\omega_{\mathrm{A}}^{(1)}$ to $\omega_{\mathrm{A}}^{(2)}$ (Fig. 1). Thus, the generated Alfvén waves will be located inside the narrow FAC region and have frequencies in the range $\omega_{\mathrm{A}}^{(1)}$ to $\omega_{\mathrm{A}}^{(2)}$. As a result, although FAC fluctuations are broadband, the resonant response of field lines inside the auroral oval selects narrowband Alfvén oscillations.

If one assumes that $j_{\|}(z)$ has maximal magnitude at the magnetospheric equator, the excited mode should have a field-aligned structure with maximum of $\Phi(z)$ at the equator (because $\left.\Phi(z) \propto j_{\|}(z)\right)$ and minimum of $b$ (because $b(z) \propto$ $\left.\partial_{z} \Phi(z)\right)$. This structure corresponds to the fundamental mode of standing Alfvén oscillations.

For a possible ground-based discrimination of the proposed FAC-associated pulsations their propagation pattern in the azimuthal direction may be considered. The KHI- and solar-wind-pressure-produced waves should demonstrate a persistent anti-sunward propagation. For FAC-related waves a wide range of apparent azimuthal propagation patterns is to be observed. Small-scale FAC fluctuations will generate, correspondingly, azimuthal small-scale background Alfvénic noise, to be screened by the ionosphere from ground magnetometers. At the same time, large-scale fluctuations will produce small- $m$ Alfvén oscillations, but without a regular propagation in the azimuthal direction.

The considered simplified 1-D model has indicated a principal possibility of the resonant ULF wave structure excitation by FAC fluctuations. In a more advanced model, an effective coupling of two oscillatory systems - KHI/solarwind-produced surface waves at the magnetopause and Alfvénic oscillations in the region of the auroral oval - may take place. These possibilities are to be examined elsewhere.

\section{Conclusions}

The specific latitudinal amplitude-phase structure of ULF waves is commonly considered as an irrefutable argument in favor of excitation of toroidal Alfvén waves by an external source (e.g., KHI of the magnetosphere boundary). Here we have shown that toroidal Pc5 Alfvén waves can be excited by fluctuating parts of the magnetospheric FAC flowing inside the auroral oval. A singularity of the obtained solution results in occurrence of a localized amplitude latitudinal peak and $180^{\circ}$ phase shift across the resonant magnetic shell, similar to the classical field line resonance. This hypothesis suggests an additional mechanism of ULF wave excitation which can operate at auroral latitudes, and it makes it possible to comprehend from a unified standpoint the reported relationships between Pc5 wave activity and the auroral oval, auroral ionospheric electroject, and magnetospheric FACs.

Acknowledgements. This study was supported by grants from the Russian Foundation for Basic Research (RFBR no. 14-05-00588; P. N. Mager), RFBR no. 15-45-05108 (V. A. Pilipenko), RSF no. 1617-00121 (O. V. Kozyreva), and US National Science Foundation AGS-1264146 (M. J. Engebretson).

The topical editor, G. Balasis, thanks A. Boudouridis and one anonymous referee for help in evaluating this paper. 


\section{References}

Agapitov, O., Glassmeier, K.-H., Plaschke, F., Auster, H.-U., Constantinescu, D., Angelopoulos, V., Magnes, W., Nakamura, R., Carlson, C. W., Frey, S., and McFadden, J. P.: Surface waves and field line resonances: A THEMIS case study, J. Geophys. Res., 114, A00C27, doi:10.1029/2008JA013553, 2009.

Bochev, A., Kudela, K., Dimitrova, I., Nenovski, P., Sinha, A., and Slivka, M.: Observations of Pc5 pulsations near fieldaligned current regions, Stud. Geophys. Geod., 53, 537-556, doi:10.1007/s11200-009-0038-2, 2009.

Cerisier, J. C., Machard, C., and Pottelette, R.: MHD turbulence generated by time-varying field-aligned currents, J. Geophys. Res., 92, 11225-11230, doi:10.1029/JA092iA10p11225, 1987.

Chen, L. and Hasegawa, A.: A theory of long period magnetic pulsation. 1. Steady state excitation of a field line resonance, J. Geophys. Res., 79, 1024-1032, doi:10.1029/JA079i007p01024, 1974.

Cheng, C. Z.: MHD field line resonances and global modes in threedimensional magnetic fields, J. Geophys. Res., 108, SMP 2-1SMP 2-8, doi:10.1029/2002JA009470, 1002, 2003.

Coult, N., Pilipenko, V., and Engebretson, M.: Suppression of resonant field line oscillations by a turbulent background, Planet. Spase Sci., 55, 694-700, doi:10.1016/j.pss.2006.03.017, 2007.

Engebretson, M., Glassmeier, K.-H., Stellmacher, M., Hughes, W. J., and Lühr, H.: The dependence of high-latitude Pc5 wave power on solar wind velocity and on the phase of highspeed solar wind streams, J. Geophys. Res., 103, 26271-26384, doi:10.1029/97JA03143, 1998.

Engebretson, M. J., Cahill, L. J., Arnoldy, R. L., Anderson, B. J., Rosenberg, T. J., Carpenter, D. L., Inan, U. S., and Eather, R. H.: The role of the ionosphere in coupling upstream ULF wave power into the dayside magnetosphere, J. Geophys. Res., 96, 1527-1542, doi:10.1029/90JA01767, 1991.

Fedorov, E., Mazur, N., Pilipenko, V., and Yumoto, K.: MHD wave conversion in plasma waveguides, J. Geophys. Res., 103, 26595 26605, doi:10.1029/98JA02578, 1998.

Fedorov, E., Pilipenko, V., and Engebretson, M.: ULF wave damping in the auroral acceleration region, J. Geophys. Res., 106, 6203-6212, doi:10.1029/2000JA000022, 2001.

Fujita, S., Glassmeier, K.-H., and Kamide, K.: MHD waves generated by the Kelvin-Helmholtz instability in a nonuniform magnetosphere, J. Geophys. Res., 101, 27317-27326, doi:10.1029/96JA02676, 1996.

Galperin, Y., Zeleny, L. M., and Kuznetsova, M.: Pinching of fieldaligned currents as a possible mechanism for the formation of raylike auroral forms, Cosmic Research, 24, 865-874, 1986.

Golovchanskaya, I. V. and Maltsev, Y. P.: On the direction of the Poynting flux related to the mesoscale electromagnetic turbulence at high latitudes, J. Geophys. Res., 109, A10203, doi:10.1029/2004JA010432, 2004.

Golovchanskaya, I. V., Ostapenko, A. A., and Kozelov, B. V.: Relationship between the high-latitude electric and magnetic turbulence and the Birkeland field-aligned currents, J. Geophys. Res., 111, A12301, doi:10.1029/2006JA011835, 2006.

Hartinger, M., Angelopoulos, V., Moldwin, M. B., Glassmeier, K.H., and Nishimura, Y.: Global energy transfer during a magnetospheric field line resonance, Geophys. Res. Lett., 38, L12101, doi:10.1029/2011GL047846, 2011.
Hartinger, M. D., Plaschke, F., Archer, M. O., Welling, D. T., Moldwin, M. B., and Ridley, A.: The global structure and time evolution of dayside magnetopause surface eigenmodes, Geophys. Res. Lett., 42, 2594-2602, doi:10.1002/2015GL063623, 2015.

Hasegawa, A., Tsui, K. N., and Assis, A. S.: A theory of long period magnetic pulsations, 3. Local field line oscillations, Geophys. Res. Lett., 10, 765-767, doi:10.1029/GL010i008p00765, 1983.

Itonaga, M., Yoshikawa, A., and Fujita, S.: A wave equation describing the generation of field-aligned current in the magnetosphere, Earth Planets Space, 52, 503-507, doi:10.1186/BF03351654, 2000.

Kessel, R. L., Mann, I. R., Fung, S. F., Milling, D. K., and O'Connell, N.: Correlation of Pc5 wave power inside and outside themagnetosphere during high speed streams, Ann. Geophys., 22, 629-641, doi:10.5194/angeo-22-629-2004, 2004.

Klimushkin, D. Yu.: Method of description of the Alfvén and magnetosonic branches of inhomogeneous plasma oscillations, Plasma Phys. Rep., 20, 280-286, 1994.

Klimushkin, D. Yu. and Chen, L.: Eigenmode stability analysis of drift-mirror modes in nonuniform plasmas, Ann. Geophys., 24, 2435-2439, doi:10.5194/angeo-24-2435-2006, 2006.

Klimushkin, D. Yu. and Mager, P. N.: The structure of lowfrequency standing Alfvén waves in the box model of the magnetosphere with magnetic field shear, J. Plasma Physics, 70, 379395, doi:10.1017/S0022377803002563, 2004.

Klimushkin, D. Yu., Mager, P. N., and Glassmeier, K.-H.: Spatiotemporal structure of Alfvén waves excited by a sudden impulse localized on an $L$ shell, Ann. Geophys., 30, 1099-1106, doi:10.5194/angeo-30-1099-2012, 2012.

Kozlovsky, A. E., Safargaleev, V. V., and Lyatsky, W. B.: The transformation of magnetoacoustic waves into Alfvén waves inside the magnetosphere, Ann. Geophys., 12, 1022-1026, doi:10.1007/s00585-994-1022-3, 1994.

Lam, H.-L. and Rostoker, G.: The relationship of Pc5 micropulsation activity in the morning sector to the auroral westward electrojet, Planet. Space Sci., 26, 473-492, doi:10.1016/00320633(78)90069-7, 1978

Leonovich, A. S.: Magnetospheric MHD response to a localized disturbance in the ionosphere, J. Geophys. Res., 105, 2507-2520, doi:10.1029/1999JA900312, 2000.

Leonovich, A. S.: A theory of field line resonance in a dipolelike axisymmetric magnetosphere, J. Geophys. Res., 106, 25803 25812, doi:10.1029/2001JA000104, 2001

Leonovich, A. S. and Mazur, V. A.: Resonance excitation of standing Alfvén waves in an axisymmetric magnetosphere (Monochromatic oscillations), Planet. Space Sci., 37, 10951108, doi:10.1016/0032-0633(89)90081-0, 1989.

Lepidi, S. and Francia, P.: Low-frequency (1-4 mHz) geomagnetic field fluctuation power at different latitudes for a diagnosis of the auroral oval position, Memorie Soc. Astron. Italiana, 74, 762765, 2003.

Mager, P. N. and Klimushkin, D. Yu.: Alfvén ship waves: high$m$ ULF pulsations in the magnetosphere generated by a moving plasma inhomogeneity, Ann. Geophys., 26, 1653-1663, doi:10.5194/angeo-26-1653-2008, 2008.

Mann, I. R., Wright, A. N., Mills, K. J., and Nakariakov, V. M.: Excitation of magnetospheric waveguide modes 
by magnetosheath flows, J. Geophys. Res., 104, 333-354, doi:10.1029/1998JA900026, 1999.

Mazur, V. A.: Resonance excitation of the magnetosphere by hydromagnetic waves incident from solar wind, Plasma Physics Reports, 36, 953-963, doi:10.1134/S1063780X10110048, 2010.

Mazur, V. A. and Chuiko, D. A.: Influence of the outermagnetospheric magnetohydrodynamic waveguide on the reflection of hydromagnetic waves from a shear flow at the magnetopause, Plasma Physics Reports, 39, 959-975, doi:10.1134/S1063780X13120064, 2013.

Menk, F. W. and Waters, C. L.: Magnetoseismology: Ground-based remote sensing of the Earth's magnetosphere, John Wiley and Sons, doi:10.1002/9783527652051, 2013.

Ohtani, S., Blomberg, L., Newell, P. T. Yamauchi, M., Potemra, T., and Zanetti, L.: Altitudinal comparison of dayside field-aligned current signatures by Viking and DMSP-F7: Intermediate-scale field-aligned current systems, J. Geophys. Res., 101, 1529715310, doi:10.1029/96JA00686, 1996.

Olson, J. V. and Rostoker, G.: Longitudinal phase variations of Pc 4-5 micropulsations, J. Geophys. Res., 83, 2481-2488, doi:10.1029/JA083iA06p02481, 1978.

Pilipenko, V. A., Watermann, J., Popov, V. A., and Papitashvili, V. O.: Relationship between auroral electrojet and Pc5 ULF waves, J. Atmos. Sol.-Terr. Phys., 63, 1545-1557, doi:10.1016/S1364-6826(01)00031-1, 2001.

Pokhotelov, O. A., Balikhin, M. A., Sagdeev, R. Z., and Treumann, R. A.: Halo and mirror instabilities in the presence of finite Larmor radius effects, J. Geophys. Res.-Space, 110, A10206, doi:10.1029/2004JA010933, 2005.

Potapov, A. S.: ULF wave activity in high-speed streams of the solar wind: Impact on the magnetosphere, J. Geophys. Res.-Space, 118, 6465-6477, doi:10.1002/2013JA019119, 2013.

Potemra, T., Zanetti, L., Bythrow, P. F., Erlandson, R. E., Lundin, R., Marklund, G. T., Block, L. P., and Lindquist, P.-A.: Resonant geomagnetic field oscillations and Birkeland currents in the morning sector, J. Geophys. Res., 93, 2661-2674, doi:10.1029/JA093iA04p02661, 1988.

Radoski, H. R.: A theory of latitude dependent geomagnetic micropulsations: the asymptotic fields, J. Geophys. Res., 79, 595613, doi:10.1029/JA079i004p00595, 1974.

Rae, I. J., Donovan, E. F., Mann, I. R., Fenrich, F. R., Watt, C. E. J., Milling, D. K., Lester, M., Lavraud, B., Wild, J. A., Singer, H. J., RèMe, H., and Balogh, A.: Evolution and characteristics of global Pc5 ULF waves during a high solar wind speed interval, Journal of Geophysical Research (Space Physics), 110, A12211, doi:10.1029/2005JA011007, 2005.

Rae, I. J., Watt, C. E. J., Fenrich, F. R., Mann, I. R., Ozeke, L. G., and Kale, A.: Energy deposition in the ionosphere through a global field line resonance, Ann. Geophys., 25, 2529-2539, doi:10.5194/angeo-25-2529-2007, 2007.

Raspopov, O. M. and Afanasieva, L. T.: Localization of the Pc5source and the auroral oval, Acta Geodaet., Geophys. et Montanist., 1792, 267-276, 1982.

Rostoker, G. and Lam, H.-L.: A generation mechanism for Pc5 micropulsations in the morning sector, Planet. Space Sci., 26, 493505, doi:10.1016/0032-0633(78)90070-3, 1978.
Saka, O., Kim, J. S., and Sugiura, M.: A cross spectral analysis of high-latitude Pc 5 pulsations in the morning sector, J. Geophys. Res., 87, 9129-9134, doi:10.1029/JA087iA11p09129, 1982.

Saka, O., Iijima, T., Yamagishi, H., Sato, N., and Baker, D. N.: Excitation of Pc 5 pulsations in the morning sector by a local injection of particles in the magnetosphere, J. Geophys. Res., 97, 10693-10701, doi:10.1029/92JA00441, 1992.

Saka, O., Hayashi, K., and Leonovich, A. S.: Ionospheric loop currents and associated ULF oscillations at geosynchronous altitudes during preonset intervals of substorm aurora, J. Geophys. Res.-Space, 120, 2460-2468, doi:10.1002/2014JA020842, 2015.

Samson, J. C.: Three-dimensional polarization characteristics of high-latitude Pc 5 geomagnetic micropulsations, J. Geophys. Res., 77, 6145-6160, doi:10.1029/JA077i031p06145, 1972.

Simms, L. E., Engebretson, M. J., Posch, J., and Hughes, W.: Effects of the equatorward auroral boundary location and solar wind parameters on Pc5 activity at auroral zone stations: A multiple regression analysis, J. Geophys. Res., 111, A10217, doi:10.1029/2005JA011587, 2006.

Southwood, D. J.: Some features of field line resonances in the magnetosphere, Planet Space Sci., 22, 483-491, doi:10.1016/00320633(74)90078-6, 1974.

Southwood, D. J. and Kivelson, M. G.: The magnetohydrodynamic response of the magnetospheric cavity to changes in solar wind pressure, J. Geophys. Res., 95, 2301-2309, doi:10.1029/JA095iA03p02301, 1990.

Sutcliffe, P. R. and Rostoker, G.: Dependence of Pc5 micropulsation power on conductivity variations in the morning sector, Planet. Space Sci., 27, 631-642, doi:10.1016/0032-0633(79)90160-0, 1979.

Tamao, T.: Transmission and coupling resonance of hydromagnetic disturbances in the non-uniform Earth's magnetosphere, Science reports of the Tohoku University, Ser. 5, Geophysics, 17, 43-72, 1965.

Vogt, J. and Haerendel, G.: Reflection and transmission of Alfvén waves at the auroral acceleration region, Geophys. Res. Lett., 25, 277-280, doi:10.1029/97GL53714, 1998.

Walker, A. D. M.: The Kelvin-Helmholtz instability in the lowlatitude boundary layer, Planet. Space Sci., 29, 1119-1133, doi:10.1016/0032-0633(81)90011-8, 1981.

Walker, A. D. M.: Excitation of field line resonances by MHD waves originating in the solar wind, J. Geophys. Res.-Space, 107, 1481, doi:10.1029/2001JA009188, 2002.

Ziesolleck, C. W. S. and McDiarmid, D. R.: Auroral latitude Pc 5 field line resonances: Quantized frequencies, spatial characteristics, and diurnal variation, J. Geophys. Res., 99, 5817-5830, doi:10.1029/93JA02903, 1994.

Zolotukhina, N. A.: Wave effects of sudden impulse and substorm onset in the magnetospheric morning sector on January 4, 2001, Geomagnetism and Aeronomy, 50, 963-969, doi:10.1134/S0016793210080062, 2010. 